\title{
Achieving universal health coverage for mental disorders
}

\author{
Vikram Patel and Shekhar Saxena discuss strategies for integrating mental disorders, and other \\ chronic conditions, into primary healthcare to achieve universal health coverage
}

$\mathrm{M}$ ental disorders are the leading contributor to the global burden of years lived with disability. ${ }^{1}$ The burden and cost of untreated mental disorders is immense for individuals, families, communities, and ultimately the world. ${ }^{2}$ A key strategy to tackling this burden is universal coverage of cost effective interventions for mental disorders, ${ }^{3}$ one target of the sustainable development goals. We analyse key questions related to this goal: why have countries failed to achieve universal health coverage for mental disorders? How can mental health interventions be integrated in primary healthcare, the foundational platform of delivery of universal health coverage? What are the lessons for integrating other chronic conditions into primary healthcare?

\section{Why have countries failed to achieve universal mental health coverage?}

In the context of mental healthcare, all countries are "developing” to some extent. Even in high income countries, the coverage gaps for common conditions like mood and anxiety disorders often exceed 50\%; in low income countries, the gap exceeds $90 \% .^{4}$ Quality gaps (a measure of the effec-

\section{REY MESSAGES}

- All countries have failed to achieve universal health coverage for mental disorders, owing to barriers related to budget and stigma

- The architecture of primary healthcare in most countries is simply not fit for the integration of mental disorders (or any chronic condition)

- Integration can be achieved using an approach that is person centred, collaborative, compassionate, engaged with the community, and that includes long term planning

- These principles could be applied across the full range of chronic conditions tiveness of the coverage) are even larger. Across all income categories, countries invest tiny fractions of their healthcare budgets on mental health, disproportionately less than the burden of mental disorders. ${ }^{5}$ This results in an inadequate number of mental health professionals per capita, a massive shortage of community based mental healthcare, and the persistence of badly run large mental hospitals.

These barriers to supply are compounded by barriers to demand, related to stigma and the discrepancies between biomedical framing of mental health problems and the conceptualisation of emotional distress in the community. Barriers to demand are one of the reasons for the large gaps in coverage observed in well resourced contexts, where universal supply of mental healthcare interventions has been largely attained-such as in the UK with its diverse mental healthcare programmes including community based mental healthcare teams and the Improving Access to Psychological Therapies programme. Further, national averages hide enormous inequities within countries, both geographic and societal-indigenous, minority, rural, and socially and economically disadvantaged communities have much poorer access to quality care. A particularly egregious example is people with severe mental disorders experiencing a loss of up to half their life expectancy relative to the general population, ${ }^{6}$ being more likely to experience homelessness and marginalisation, and being denied the basic rights to freedom and dignity through incarceration in hospitals or prisons.

Since the Alma Ata declaration in 1978, the means of improving access to mental healthcare has been to integrate it with primary healthcare. But after four decades of trying, we know that achieving such integration at scale will require nothing short of a wholescale re-engineering of the healthcare system. At the heart of the challenge is the architecture of primary healthcare in most countries, which is simply not fit for the integration of mental disorders (or any chronic condition). ${ }^{8}$ Historically, primary healthcare was for acute or episodic medical events-from cuts to colds, extending to childbirth and the management of acute infections such as malaria and diarrhoea. Anything more complex (involving long term care or requiring a person centred approach to care, beyond a reductionist biomedical diagnosis) was either ignored or passed to secondary care. Attempts to integrate mental health have failed because they have not tackled these fundamental barriers; instead, they have tried to replicate secondary care in primary carefor example, by posting psychiatrists in primary healthcare centres, ${ }^{910}$ a strategy that is neither scalable nor necessary.

\section{How can mental healthcare be integrated into primary care?}

A key element of the field of global mental health is the design and evaluation of innovative strategies for integrating cost effective pharmacological and psychosocial interventions in primary healthcare. ${ }^{111}$ The evidence from this work, from a range of contexts including high income countries, is showing the way to integration. ${ }^{1} \mathrm{~A}$ theme across this evidence is the placement of non-specialised providers (including peers, community health workers, and nurses) in primary healthcare and community settings to perform diverse roles such as coordinating collaborative care ${ }^{12}$; educating and mobilising the community to increase demand for care ${ }^{13}$; supporting families and patients to tackle proximal social determinants of mental health; and delivering empirically supported psychological and social interventions. ${ }^{14}$

The growing recognition that binary models of diagnosis of mental disorders do not capture the dimensional distribution of symptoms, distress, and disability of mental health problems in the population has important implications for treatment planning. A “one size fits all” approach does not work. Instead, we need a staged approach whereby interventions 
are delivered based on both symptom severity and the effect of these symptoms on distress and disability. ${ }^{15}$ This aligns with the notion that most care targeting relatively mild, early, or transient stages of mental distress can be delivered through self care and by non-specialist providers with appropriate skills. This facilitates quicker recovery for those people while simultaneously identifying those who might need more intensive interventions for referral to specialised providers. This approach, which reduces the emphasis on biomedical diagnoses, is also more likely to be acceptable to the wider population and to be less stigmatising.

The exponential growth of digital health innovations-spanning guided self care, training and supervision of frontline workers, remote consultations by specialist providers, and remote monitoring of mental health-offers a transformative opportunity to bypass historical structural barriers to enabling task sharing and collaborative care. ${ }^{16}$ Several examples of innovative programmes and projects can be found in the Mental Health Innovation Network (www.mhinnovation.net) and the Lancet Commission on Global Mental Health and Sustainable Development. ${ }^{17}$

We have identified five key elements needed to integrate mental health in primary care, which we refer to as the "5C approach" (box 1). Although these elements were derived from innovations seeking to attain universal coverage of mental healthcare, they can be applied to the full range of chronic conditions.

Three key points need to be emphasised. First, integration must cover the full range of mental disorders, in particular ensuring that people with severely disabling conditions-such as schizophrenia, alcohol and drug dependence, and dementiaare not left behind as they are less likely to seek care, less likely to use digital technology, and more likely to experience discrimination, isolation, and premature mortality. Second, coverage must be equity sensitive, recognising that subgroups in the population that experience higher levels of deprivation or exclusion, such as poor people, refugees or ethnic, religious, or sexual minorities, bear a disproportionate burden of mental disorders. Third, integration must emphasise quality of care for both the mental disorder (for example, to abolish coercive, harmful, and abusive practices) and co-existing physical health conditions, which are major contributors to premature mortality.

\section{What are the lessons for integrating chronic conditions?}

Mental healthcare has led the development of care strategies for health conditions characterised by a chronic, episodic, or relapsing course. At the heart of these innovations is the transition of delivery of long term care from institutions to the community, with the goal of decreasing disabilities, optimising quality of life, slowing disease progression, and minimising the risk of relapse. Interventions have focused on "recovery" by going beyond the specific symptoms of the disorder to tackle impairments in daily life and experiences prioritised by the patient-the hallmark of person centred care.

Mental health programmes have championed the integration of pharmacological with psychological and social interventions, referred to as the biopsychosocial approach to care; the engagement of family members (where culturally appropriate and agreed with the patient) to support recovery and tackling the needs of caregivers; and intersectoral interventions to promote the inclusion of people with mental disabilities and

\section{Box 1: The " 5 C" approach to integrating mental health in universal health coverage}

- Person centred-focusing on what matters to the patient rather than what is the matter with the patient, ${ }^{21}$ which translates into attending to functional needs, multiple morbidities, and social suffering and to the empowerment of the person to harness their personal and community resources to enable recovery with dignity

- Continuing or long term planning-recognising that "cures" are rare and the goal of care is to optimise the quality of life and health

- Community platform of delivery-engagement with families and the broader community to tackle stigma, adherence, and other barriers to the uptake of effective care ${ }^{1}$

- Collaborative care-with seamless coordination by community health workers or case managers of primary care and specialist providers to ensure high coverage of quality care and early "stepping up" of the intensity of care when needed ${ }^{12}$

- Compassionate stance-instils hope, a key ingredient for patient engagement, motivates health promoting behaviours, and harnesses the placebo effect (which has a robust neuroscientific basis) ${ }^{22}$ to promote mental health. Examples of innovative delivery strategies include using non-specialist providers, including peers, to deliver psychosocial interventions, ${ }^{14}$ using digital platforms to support guided self care and training and supervision of providers, ${ }^{16}$ and using collaborative care with case managers to manage multiple morbidities. ${ }^{1218}$ Engaging civil society to increase the demand for care, to tackle stigma and discrimination, and to design, deliver, and hold services accountable has helped reduce barriers to demand while also empowering people with lived experience.

Despite robust evidence from pilot studies and trials, little progress has been made in scaling up these strategies in most countries. ${ }^{19}$ Major barriers that remain include financing of non-specialist providers to deliver psychosocial interventions; implementing scalable approaches to training, supervision, support, and quality assurance; and institutionalising collaborative and coordinated care. The goal of improving the recognition of mental disorders and delivery of mental health interventions in primary care remains a distant one for most of the world.

This focus on implementation science is at the heart of the work of the PRIME consortium $^{20}$ (sponsored by the UK Department for International Development) and the National Institute of Mental Health's research partnerships for scaling up mental health interventions in low and middle income countries (https://www. nimh.nih.gov/about/organization/cgmhr/ scaleuphubs/index.shtml). The goal is to show how routine healthcare systems can fully integrate the strategies that have proved effective for the management of mental disorders. A key strategy is integration of care with other chronic conditions. Mental disorders (including substance use disorders), for example, worsen the outcomes of cardiovascular and metabolic disorders, which are major contributors to the premature mortality of people with severe mental disorders. ${ }^{18}$ The synergies between non-communicable diseases and mental health problems are recognised in inclusion of mental health in the scope of the World Health Organization's independent high level commission and the United Nations' high level meeting on noncommunicable diseases.

\section{Conclusions}

As the global health community reflects on the role of primary healthcare in this 40th anniversary of the historic Alma Ata declaration, we reaffirm the view that pri- 
mary healthcare must be the foundation of the architecture of universal health coverage to realise the goals of reducing the unmet need for mental healthcare globally. Achieving this, however, will require fundamental re-engineering of the way that primary healthcare is conceptualised, organised, and delivered, and this, in turn, will need the full engagement and support of all actors in universal healthcare, not least people who are affected by mental disorders. The rising burden of mental disorders, in all countries, requires immediate and dramatic actions, informed by the rich body of evidence on delivery innovations from diverse contexts. Failure to do so will mean failure to achieve universal health coverage-universal refers not just to coverage of the population but also coverage of the full range of its health needs, and health should be considered comprehensively, to include mental health and social wellbeing alongside physical health.

Contributors and sources: Both authors came up with the concept of the article. VP led the drafting and $\mathrm{SS}$ reviewed, edited, and contributed to the draft. Both authors have approved the revision.

Competing interests: Neither author has any conflicts of interest to disclose. VP is supported by National Institute of Mental Health and the UK Department for International Development grants.

Provenance and peer review: Commissioned; externally peer reviewed.

This article is part of a series commissioned by The $B M J$ based on an idea from the Harvard Global Health Institute. The BM/ retained full editorial control over external peer review, editing, and publication. Harvard Global Health Institute paid the open access fees. Vikram Patel, Pershing Square professor of global health and Wellcome Trust principal research fellow Shekhar Saxena, professor of practice Harvard TH Chan School of Public Health, USA Correspondence to: VPatel vikram_patel@hms.harvard.edu

\section{(c) $\underset{\mathrm{Br}}{(1)}$ (8) OPEN ACCESS}

This is an Open Access article distributed in accordance with the Creative Commons Attribution
Non Commercial (CC BY-NC 4.0) license, which permits others to distribute, remix, adapt, build upon this work non-commercially, and license their derivative works on different terms, provided the original work is properly cited and the use is non-commercial. See: http://creativecommons.org/ licenses/by-nc/4.0/.

\section{Check for updates}

1 Patel V, Chisholm D, Parikh R, et al, DCP MNS Author Group. Addressing the burden of mental, neurological, and substance use disorders: key messages from Disease Control Priorities, 3rd edition. Lancet 2016;387:1672-85. doi:10.1016/ S0140-6736(15)00390-6

2 Chisholm D, Sweeny K, Sheehan P, et al. Scalingup treatment of depression and anxiety: a global return on investment analysis. Lancet Psychiatry 2016;3:415-24. doi:10.1016/S22150366(16)30024-4

3 Chisholm D, Naci H, Hyder AA, Tran NT, Peden M. Cost effectiveness of strategies to combat road traffic injuries in sub-Saharan Africa and South East Asia: mathematical modelling study. BMJ 2012;344:e612. doi:10.1136/bmj.e612

4 Thornicroft G, Chatterii S, Evans-Lacko S, et al. Undertreatment of people with major depressive disorder in 21 countries. $\mathrm{Br} \mathrm{J}$ Psychiatry 2017;210:119-24. doi:10.1192/bjp. bp.116.188078

5 World Health Organization. Mental Health ATLAS 2014. WHO, 2015.

6 Walker ER, McGee RE, Druss BG. Mortality in mental disorders and global disease burden implications: a systematic review and meta-analysis. JAMA Psychiatry 2015;72:334-41. doi:10.1001/ jamapsychiatry.2014.2502

7 Kleinman A. Global mental health: a failure of humanity. Lancet 2009;374:603-4. doi:10.1016/ S0140-6736(09)61510-5

8 Beaglehole R, Epping-Jordan J, Patel V, et al. Improving the prevention and management of chronic disease in low-income and middle-income countries: a priority for primary health care. Lancet 2008;372:940-9. doi:10.1016/S01406736(08)61404-X

9 Gilbody S, Whitty P, Grimshaw J, Thomas R. Educational and organizational interventions to improve the management of depression in primary care: a systematic review. JAMA 2003;289:3145-51. doi:10.1001/jama.289.23.3145

10 van Ginneken N, Maheedhariah MS, Ghani S, Ramakrishna J, Raja A, Patel V. Human resources and models of mental healthcare integration into primary and community care in India: Case studies of 72 programmes. PLoS One 2017;12:e0178954. doi:10.1371/journal. pone.0178954
11 Collins PY, Saxena S. Action on mental health needs global cooperation. Nature 2016;532:25-7. doi:10.1038/532025a

12 Patel V, Belkin GS, Chockalingam A, et al. Integrating mental health services into priority health care platforms: addressing a grand challenge in global mental health. PLoS Med 2013;10:e1001448. doi:10.1371/journal. pmed.1001448

13 Shidhaye R, Murhar V, Gangale S, et al. The effect of VISHRAM, a grass-roots community-based mental health programme, on the treatment gap for depression in rural communities in India: a population-based study. Lancet Psychiatry 2017;4:128-35. doi:10.1016/S22150366(16)30424-2

14 Singla DR, Kohrt BA, Murray LK, Anand A, Chorpita BF, Patel V. Psychological treatments for the world: lessons from low- and middle-income countries. Annu Rev Clin Psychol 2017;13:149-81. doi:10.1146/annurev clinpsy-032816-045217

15 Patel V. Talking sensibly about depression. PLoS Med 2017;14:e1002257. doi:10.1371/journal. pmed.1002257

16 Naslund JA, Aschbrenner KA, Araya R, et al. Digital technology for treating and preventing mental disorders in low-income and middle-income countries: a narrative review of the literature. Lancet Psychiatry 2017;4:486-500. doi:10.1016/S22150366(17)30096-2

17 Patel V, Saxena S, Lund C, et al. The Lancet Commission on global mental health and sustainable development. Lancet 2018;392:1553-98 doi:10.1016/S0140-6736(18)31612-X

18 Patel V, Chatterii S. Integrating mental health in care for noncommunicable diseases: an imperative for person-centered care. Health Aff (Millwood) 2015;34:1498-505. doi:10.1377/ hlthaff.2015.0791

19 Eaton J, McCay L, Semrau M, et al. Scale up of services for mental health in low-income and middleincome countries. Lancet 2011;378:1592-603. doi:10.1016/S0140-6736(11)60891-X

20 Lund C, Tomlinson M, De Silva M, et al. PRIME: a programme to reduce the treatment gap for mental disorders in five low- and middle-income countries. PLoS Med 2012;9:e1001359. doi:10.1371/journal. pmed.1001359

21 Kebede S. Ask patients "What matters to you?" rather than "What's the matter?"BMJ 2016;354:14045. doi:10.1136/bmj.i4045

22 Geuter S, Koban L, Wager TD. The cognitive neuroscience of placebo effects: concepts, predictions, and physiology. Annu Rev Neurosci 2017;40:167-88. doi:10.1146/annurev neuro-072116-031132

Cite this as: $B M J$ 2019;366:14516 http://dx.doi.org/10.1136/bmj.l4516 